\title{
Determinação dos diagnósticos de depressão, tentativa de suicídio, gravidez, vírus da imunodeficiência humana (HIV) e doenças sexualmente transmissíveis (DST) em adolescentes e adultos jovens
}

\author{
Determination of the diagnoses of depression, suicide attempts, \\ pregnancy, human immunodefficiency virus (HIV), and sexually \\ transmitted diseases (STDs) in adolescents and young adults
}

\author{
Sandra Odebrecht Vargas Nunes ${ }^{1}$; Rosemarie Elizabeth de Abreu²; Ana Lúcia \\ Hirata $^{3}$; Mariane Vargas Alves Nunes ${ }^{4}$; Renata Merli Franco ${ }^{4}$; Luisa Raizer Barbosa ${ }^{4}$
}

Resumo

O objetivo deste trabalho foi determinar a freqüência dos diagnósticos de Depressão, Tentativas de Suicídio, Doenças Sexualmente Transmissíveis (DST), HIV (Vírus da Imunodeficiência Humana) e Gravidez; coletados de 2523 prontuários de adolescentes nas idades de 10 a 16 anos e de 17 a 25 anos, atendidos no Hospital Universitário (HU/UEL). De 10 a 16 anos, os diagnósticos e identidade de gênero foram: Depressão (6 feminino, 8 masculino), tentativas de suicídio ( 2 feminino, 2 masculino), HIV ( 2 feminino, 1 masculino), DST ( 8 feminino, 1 masculino), Gravidez ( $\mathrm{n}=227)$. Nas idades de 17 a 25 anos os diagnósticos e a identidade de gênero foram: Depressão (46 feminino, 7 masculino), tentativas de suicídio (11 feminino, 2 masculino), DST (90 feminino, 22 masculino), HIV (19 feminino, 9 masculino) e gravidez ( $\mathrm{n}=2074)$. Os resultados sugerem o aumento na freqüência dos diagnósticos estudados nas idades de 17 a 25 anos. As condutas de risco iniciaram-se precocemente, por volta de 10 a 16 anos. Sugerem-se intervenções que privilegiem o aumento da auto-estima e dêem atenção em prevenção pela educação e detecção precoce, que antecede o ensino médio.

Palavras-chave: Adolescência. Gravidez. Depressão. Tentativa de suicídio. Doenças Sexualmente Transmissíveis.

\begin{abstract}
The purpose of this work was to determine the frequency of diagnoses of depression, suicide attempts, Sexually Transmitted Diseases (STDs), Human Immunodefficiency Virus (HIV) and pregnancy, in data collected from 2523 medical records of adolescents between the ages of 10 to 16 years old, and 17 to 25 years old, attended at University Hospital (University Hospital / State University of Londrina). Between the ages of 10 to 16 years old, the diagnoses and gender identification were: depression (6 female, 8 male); suicide attempts ( 2 female, 2 male); HIV ( 2 female, 1 male); STDs ( 8 female, 1 male); and pregnancy $(\mathrm{n}=227)$. As for the ages of 17 to 25 years old, the diagnoses and gender identification were: depression (46 female, 7 male); suicide attempts (11 female, 2 male); HIV (19 female, 9 male); STDs (90 female, 22 male); pregnancy $(n=2074)$. These results suggest the increase of studied diagnoses at ages ranging from 17 to 25 . Risk behavior started prematurely, around the ages of 10 to 16 years old. These results suggest the use of interventions that increase self-esteem and give proper attention to prevention by means of education and early detection, prior to high school.

Key words: Adolescence. Pregnancy. Suicide attempts. Sexually Transmitted Diseases (STDs).

1 Docente do Departamento de Clínica Médica da Universidade Estadual de Londrina (UEL-PR).

2 Docente do Departamento de Fundamentos de Psicologia e Psicanálise da Universidade Estadual de Londrina (UEL-PR).

3 Bolsista de Iniciação Científica PIBIC/CNPq da Universidade Estadual de Londrina (UEL-PR).

4 Acadêmicas do Curso de Medicina da Universidade Estadual de Londrina (UEL-PR)
\end{abstract}




\section{Introdução}

A adolescência é um período de grandes riscos e oportunidades. As dramáticas mudanças biológicas que acompanham essa fase de transição são essencialmente as mesmas, mas o contexto social em que elas ocorrem estão diferentes, iniciando-se mais precocemente e as mudanças acontecem mais rapidamente (MILLSTEIN et al., 1993).

Nesse contexto, apesar de os educadores e profissionais da área de saúde desenvolverem conhecimentos e atividades precoces, visando a auxiliar os adolescentes a tomar decisões em favor da promoção de saúde e adotar um estilo de vida saudável, a depressão, as tentativas de suicídio, as doenças sexualmente transmissíveis (DST) e a Síndrome da imunodeficiência adquirida (AIDS) estão ocorrendo cada vez mais precocemente, incidindo a partir do início da adolescência em relação direta com o aumento de gravidez precoce (OMS, 1977).

Devido às repercussões sobre a mãe e o concepto, a gestação na adolescência é considerada de alto risco pela Organização Mundial da Saúde. Porém, acredita-se atualmente que o risco seja mais social do que biológico (OMS, 1977).

O início das experiências sexuais cada vez mais cedo, a multiplicidade de parceiros e o não-uso de preservativos nas relações sexuais têm sido apontados como fatores de risco, e são influenciados por um sistema de gênero que se pauta na dominação masculina (TAQUETTE; VILHENA; PAULA, 2004).

Quando a atividade sexual tem como resultante a gravidez, gera conseqüências na vida tanto da adolescente, quanto do recém-nascido. A adolescente poderá apresentar problemas de crescimento e desenvolvimento, emocionais e comportamentais, educacionais e de aprendizado, além de complicações da gravidez e problemas de parto (CREATSAS et al., 1991; PIYASIL, 1998; WILCOX; FIELD, 1998).

O aumento da incidência de DSTs em adolescentes está ocorrendo no mesmo período em que surge o crescimento da gravidez na adolescência. Estima-se que no Brasil, a cada ano, um milhão de adolescentes entre 10 e 20 anos dão à luz, o que corresponde a $20 \%$ dos nascidos vivos (DUARTE, 1998).

Os estudos têm demonstrado maiores riscos na gravidez de adolescentes em relação a mulheres mais velhas como: prematuridade, baixo peso ao nascer, morbidade e mortalidade perinatal (BLANKSON et al., 1993).

Observa-se que, na etapa adolescente, as relações sexuais iniciam-se mais cedo e com maior número de parceiros, o que contribui para aumentar a ocorrência das DSTs. Entre adolescentes o uso de preservativos é baixo e a atividade sexual não é programada. Estudos brasileiros revelam que apenas um terço deles ou menos usam preservativo em todas as relações sexuais. Segundo dados de pesquisa divulgados pelo Ministério da Saúde, os mais baixos índices de uso se encontram entre 15 e 19 anos. No Brasil não há informações sobre a prevalência de DSTs entre adolescentes, as únicas de notificação compulsória são a sífilis e a AIDS. Além disto, cerca de $70 \%$ das pessoas com alguma DST buscam tratamento em farmácias, o que faz com que o número de casos notificados fique abaixo da estimativa (TAQUETTE; VILHENA; PAULA, 2004).

Do total de casos de AIDS, segundo as categorias de exposição, a via de transmissão sexual é a predominante, cerca de $50 \%$. De acordo com o Ministério da Saúde, o maior número de notificações acumuladas entre 1980 e 1999, 67.267 casos, ou seja, $43,23 \%$ do total concentra-se entre 15 e 24 anos. Como o tempo de latência da doença é longo, chegando até 11 anos, podemos inferir que grande parte destes jovens deve ter-se infectado na adolescência (TAQUETTE; VILHENA; PAULA, 2004).

Sabroza et al. (2004) ressaltam que a gravidez na adolescência aumenta o risco de repercussões emocionais negativas e situações de baixa auto- 
estima, assim como reação negativa da família, menor valorização e expectativa em relação ao futuro e grande sofrimento psíquico.

Dada a sua imaturidade e labilidade emocional, podem ocorrer importantes alterações psicológicas, gerando extrema dificuldade em adaptar-se à sua nova condição, exacerbando sentimentos que já estavam presentes antes da gravidez, como a depressão (FRIEDMAN; PHILLIPS, 1981).

A depressão maior na adolescência é comum, debilitante, e recorrente, envolvendo um alto grau de morbidade e mortalidade e um dos maiores problemas de saúde pública, embora não diagnosticada e nãotratada (BAHLS, 2002).

$\mathrm{Na}$ faixa etária de adolescentes e adultos jovens, a depressão tem uma prevalência de $16,8 \%$, sendo uma doença incapacitante para o trabalho, habilidades educacionais e interações sociais. Visto que os adolescentes e adultos jovens estão em uma fase chave de socialização em termos de carreira profissional e relações interpessoais, esse índice indica um considerável risco potencial para complicação e futuro risco de cronicidade do estado de depressão (WITTCHEN; NELSONC; LACHNER, 1998).

Pacientes com transtornos depressivos têm risco de $15 \%$ de suicídio, sendo esta, entre adolescentes dos 15 aos 24 anos, a segunda causa de morte. Reforçando este dado, Beautrais (2003) informa que as taxas de suicídio em jovens entre 15-24 anos de idade duplicaram no período de 1985 a 1995. O risco de suicídio é alto entre os adolescentes, porque nesta faixa etária eles são emocionalmente lábeis e impulsivos, sendo esta uma combinação letal. Por este motivo, todos os profissionais de saúde devem avaliar este risco nos adolescentes, visto que dois terços destes que cometeram suicídio foram vistos por seus médicos um mês antes de suas mortes (HIRSCHFELD; RUSSELL, 1997).

Depressão maior é uma das doenças psiquiátricas mais comuns na adolescência e suas muitas conseqüências podem incluir morbidade para a saúde mental e abuso de substâncias psicoativas, prejuízo acadêmico e social, gravidez não planejada e altas taxas de tentativa de suicídio (MARTIN; DONALD, 2000).

Lewinsohn et al. (2000) argumentam que adolescentes que desenvolveram problemas psiquiátricos quando se tornaram adultos jovens, são caracterizados durante a adolescência por terem episódios depressivos mais severos (episódios longos, múltiplos; maior número de sintomas; história de tentativa de suicídio).

Era de se esperar que a gravidez atuasse como um evento de vida estressante e estaria associado com depressão em adolescentes pessimistas. Entretanto, estudos comprovaram que adolescentes grávidas com personalidade pessimista tiveram menos depressão que adolescentes não deprimidas. Entretanto, após o nascimento de seus filhos, as pacientes pessimistas apresentaram mais depressão que as não pessimistas (WAGNER et al., 1998).

O estudo propõe avaliar a freqüência de depressão, tentativas de suicídio, doenças sexualmente transmissíveis (DST) e AIDS, na adolescência e adultos jovens, assim como suas possíveis e suas relações com a gravidez nesta população.

Para prevenir a gravidez em adolescentes, deverá ocorrer uma intervenção precoce educativa iniciada nas idades escolares, ou seja, no ensino fundamental. A conscientização para prevenir as condutas de risco ainda é a melhor solução e deve ser iniciada nos primeiros anos de vida por meio da educação proporcionada pelos pais, escola e comunidade.

\section{Métodos}

O trabalho refere-se a uma análise descritiva obtida através de prontuários 2523 prontuários do Hospital Universitário, da Universidade Estadual de Londrina (HU/UEL) no período de um ano (20022003), referentes a pacientes atendidos com diagnósticos de DSTs, HIV, depressão, gravidez e tentativa de suicídio. Os pacientes com os 
diagnósticos foram divididos em dois grupos distintos: 1) 10 a 16 anos e o 2) 17 a 25 anos.

Os dados coletados para esta análise foram os do período de setembro de 2002 a setembro de 2003. $\mathrm{Na}$ população estudada foram consideradas duas fases distintas: a primeira de dez aos dezesseis anos e a segunda, dos dezessete aos vinte e cinco anos.

Os dados foram levantados em prontuários através dos diagnósticos da décima Revisão da Classificação Internacional de Doenças - Décima edição (CID10), da Organização Mundial de Saúde (OMS). E os diagnósticos de tentativas de suicídio foram os cadastrados para notificação obrigatória para órgãos públicos.

Foram levantados dados de 2523 prontuários de adolescentes nas idades de 10 a 16 anos, e de 17 a 25 anos; atendidos no Hospital Universitário (HU/ UEL). De 10 a 16 anos, os diagnósticos e identidade de gênero foram: depressão $n=14$, tentativas de suicídio $\mathrm{n}=4, \operatorname{HIV} \mathrm{n}=3$, DST $\mathrm{n}=9$, Gravidez $\mathrm{n}=227$. Nas idades de 17 a 25 anos, os diagnósticos e a identidade de gênero foram: depressão $n=53$, tentativas de suicídio $n=13$, DST $n=112, \operatorname{HIV} n=28$ e gravidez $n=2074$, podendo constar em cada prontuário mais de um diagnóstico.

O banco de dados foi organizado em planilhas do Programa Excel-Windows 98, o que possibilitou a organização dos dados em gráficos.

O estudo foi aprovado pelo Comitê de ética em Pesquisa da UEL, ofício 107/02

\section{Resultados}

As variáveis analisadas foram: os diagnósticos dos prontuários médicos, a identidade de gênero e a distribuição da faixa etária.

Nas idades de 10 a 16 anos, os diagnósticos e identidade de gênero foram: episódio depressivo ou transtorno depressivo recorrente $(\mathrm{n}=14 ; 6$ Feminino, 8 Masculino), tentativas de suicídio ( $\mathrm{n}=4 ; 2$ Feminino, 2 Masculino), HIV ( $n=3 ; 2$ Feminino, 1 Masculino),
DST ( $\mathrm{n}=9$; 8 Feminino, 1 Masculino), Gravidez $(\mathrm{n}=227)$. Nas idades de 17 a 25 anos, os diagnósticos e a identidade de gênero foram: depressão ( $n=53 ; 46$ Feminino, 7 Masculino), tentativas de suicídio ( $\mathrm{n}=$ 13; 11 Feminino, 2 Masculino), DST ( $\mathrm{n}=112 ; 90$ Feminino, 22 Masculino), HIV ( $\mathrm{n}=28$; 19 Feminino, 9 Masculino) e gravidez $(\mathrm{n}=2074)$.

O Gráfico 1 demonstrou maior freqüência do sexo feminino nos casos atendidos por depressão, tentativa de suicídio, DST, HIV na faixa etária de 10 a 25 anos. Dos 64 casos de depressão atendidos, 53 foram do sexo feminino e 11 do masculino. Dos 17 casos de tentativa de suicídio, 14 foram do sexo feminino e 3 do masculino. Dos 31 casos de HIV, houve 21 casos femininos e 10 casos masculinos. Dos 121 casos de DST, houve 98 casos do sexo feminino e 23 casos do masculino.

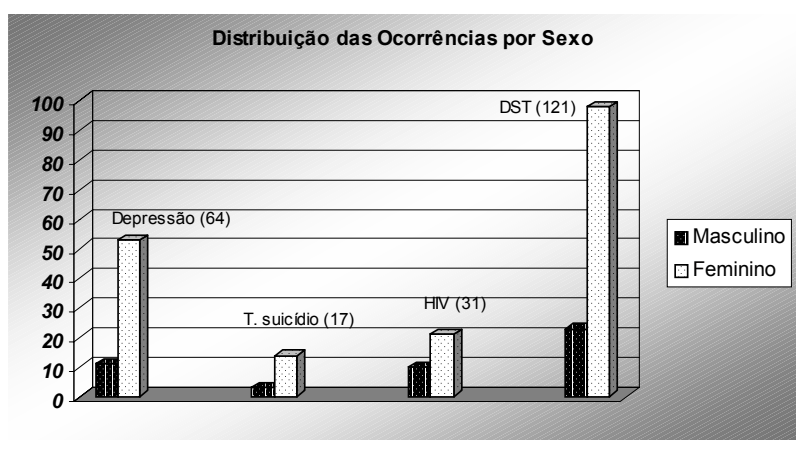

Gráfico 1. Freqüência de depressão, tentativa de suicídio, HIV, DST por gênero.

As distribuições da faixa etária de tentativas de suicídio registradas foram de 2 casos em cada uma das idades: 11, 19, 21, 22 e 24 anos, representado 11,76\% cada. Aos 13, 16, 18 e 23 anos foram registrados apenas um caso por faixa etária, correspondendo a 5,88\% cada. O maior registro (3 casos) foi aos 25 anos, correspondendo a 17,65\%.

Na população diagnosticada com DST, foi encontrado no total entre 14 e 15 anos, apenas 1 caso em cada faixa etária, representando $0,82 \%$ individualmente. Na idade de 16 anos, houve 7 casos registrados, correspondendo a 5,7\%. Aos 17 e 23 anos, 10 casos $(8,2 \%)$ cada. Aos 18 anos, 9 casos 
(7,4\%). Aos 19 anos, 16 casos (13,2\%). Aos 20 e 22 anos, 17 casos cada (14\%). Aos 21 anos, 14 casos $(11,5 \%)$. Aos 24 anos houve um total de 11 casos (9\%). Aos 25 anos, 8 casos (6,6\%).

Nos casos de depressão, os primeiros relatados foram aos 11 anos (3 casos, 4,6\%). Essa porcentagem apresentou-se igual aos 16 e 21 anos. Não houve relatos aos 10, 13 e 14 anos. Aos 12 anos foi registrado 1 caso (1,5\%). Nas idades de 15, $17 \mathrm{e}$ 25 anos registraram-se 4 casos em cada idade (6,2\%). Na faixa etária de 18 e 22 anos, houve 7 casos relatados em cada (10,9\%). Aos 19 e 20 anos, 6 casos em cada (9,3\%). Aos 24 anos, 5 casos (7,8\%). Aos 23 anos correspondendo à maior incidência, 11 casos $(17,19 \%)$.

Nos pacientes diagnosticados com HIV, registraram-se casos entre 10 e 12 anos reaparecendo novos casos aos 17 anos. Não houve casos registrados nas idades de 11, 13, 14, 15 e 16 anos.

As maiores ocorrências de HIV foram nas idades de 19, 24 e 25 anos, representando 5 casos cada (16,13\%). Aos 10, 17 e 23 anos, 2 casos em cada $(6,45 \%)$. Aos 12, 18 e 21 anos, 1 caso em cada (3,23\%). Aos 20 anos, 3 casos em cada (9,68\%). Aos 22 anos, 4 casos (12,9\%).

No Gráfico 2, foram avaliados 2301 prontuários de adolescentes grávidas, com faixa etária de 13 anos em diante. A distribuição por idade foi respectivamente: 13 anos $(n=4) ; 14$ anos $(n=22) ; 15$ $\operatorname{anos}(\mathrm{n}=86) ; 16$ anos $(\mathrm{n}=115) ; 17$ anos $(\mathrm{n}=164) ; 18$ $\operatorname{anos}(\mathrm{n}=180) ; 19$ anos $(\mathrm{n}=224) ; 20$ anos $(\mathrm{n}=268) ; 21$ $\operatorname{anos}(\mathrm{n}=253) ; 22$ anos $(\mathrm{n}=259) ; 23$ anos $(\mathrm{n}=271) ; 24$ $\operatorname{anos}(n=220)$ e 25 anos $(n=235)$.

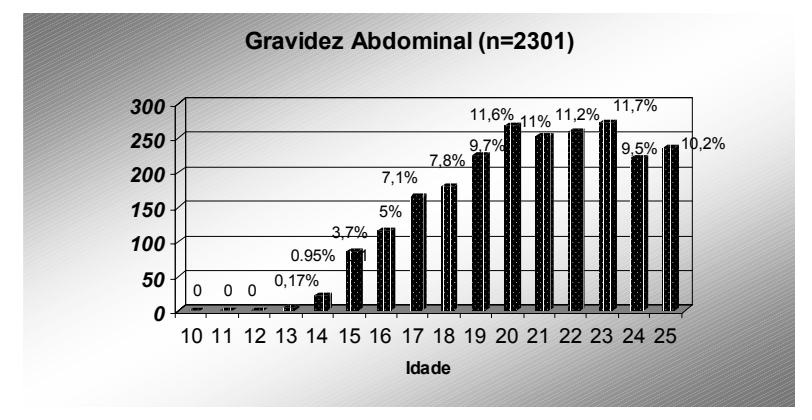

Gráfico 2. Freqüência dos casos de gravidez na faixa etária de 10 a 25 anos.

Verificaram-se 227 casos na faixa etária entre 10 a 16 anos, enquanto na faixa etária dos 17 aos 25 anos, 2.074 casos.

Os gráficos 3, 4 e 5 avaliaram a correlação da gravidez com DST, HIV e depressão respectivamente.

No gráfico 3, avaliou-se a correlação entre DST e gravidez na faixa etária de 10 a 25 anos. Nos 121 casos analisados, $56(46 \%)$ destas pacientes com DST eram gestantes. Os fatores que predispõem à gravidez podem ser os mesmos que predispõem às DSTs em adolescentes e adultos jovens.

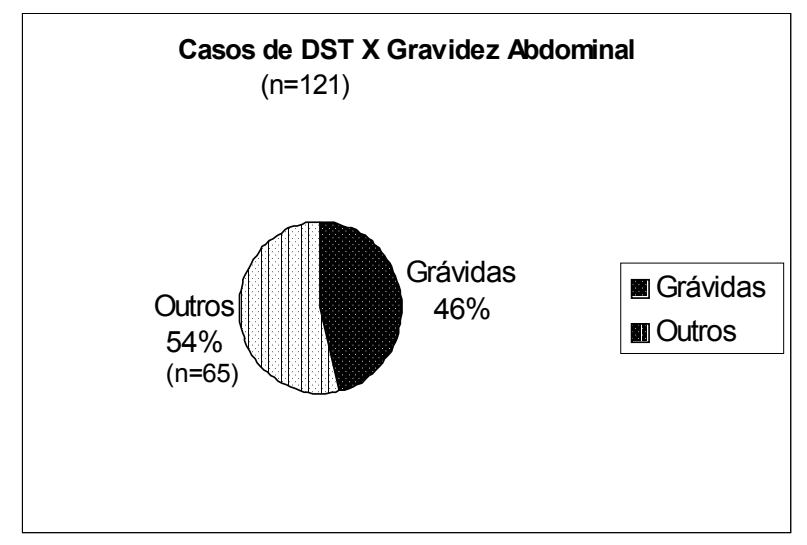

Gráfico 3. Correlação entre DSTs e gravidez na faixa etária de 10 a 25 anos.

No gráfico 4, avaliou-se a correlação entre HIV e gravidez na faixa etária de 10 a 25 anos.

Dos 31 casos de HIV, 6 casos (19\%) de pacientes diagnosticadas com infecção por HIV eram gestantes. 
Casos de HIV X Gravidez Abdominal

$(n=31)$

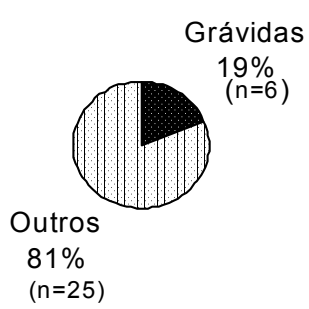

Grávidas

Outros

faixa etária entre 10 aos 25 anos.

O gráfico 5 apresenta a correlação entre depressão e gravidez na faixa etária de 10 a 25 anos. Nos 64 casos de depressão, 12 casos (19\%) de pacientes diagnosticadas por episódio depressivo ou transtorno depressivo recorrente, de acordo com os critérios diagnósticos da CID-10, estavam grávidas na época do diagnóstico.

Casos de Depressão X Gestação $(n=64)$

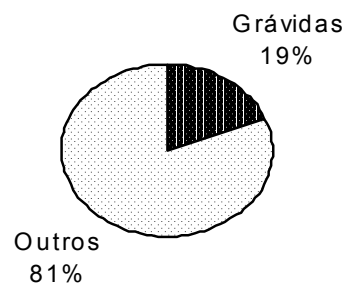

Grávidas $\square$ Outros

$81 \%$

Gráfico 5. Correlação entre depressão e gestação na faixa etária de 10 a 25 anos.

\section{Discussão}

No estudo, $46 \%$ dos casos relatados de DST ocorreram em gestantes coincidindo com a literatura revisada. Segundo Duarte (1998), a incidência de DST em adolescente está aumentando proporcionalmente ao crescimento da gravidez na adolescência.

Do total de 64 casos diagnosticados por episódio depressivo ou transtorno depressivo recorrente neste estudo, 12 pacientes (19\%) estavam grávidas na época do diagnóstico. Isso pode ser explicado por sua imaturidade e labilidade emocional que podem ocasionar importantes alterações psicológicas, gerando extrema dificuldade em adaptar-se à sua nova condição, exacerbando sentimentos que já estavam presentes antes da gravidez, como a depressão (FRIEDMAN; PHILLIPS, 1981).

No presente estudo, encontraram-se pacientes gestantes em 19\% dos diagnósticos com infecção por HIV. Isso pode ser explicado pelo baixo índice de uso de preservativos durante a adolescência, o que predispõe tanto à gravidez quanto à infecção por HIV. Segundo Taquette, Vilhena e Paula (2004), um terço ou menos dos adolescentes usam preservativos sempre.

Não foram relatados casos de tentativas de suicídio em gestantes. Isso pode ser devido ao pequeno número de casos relatados em nosso serviço durante o período de 1 ano.

Krug et al. (2002) salientam que o ambiente social inadequado associado ao abuso sexual e negligência na infância resulta em aumento na adolescência de: gravidez, DST, HIV, depressão e tentativas de suicídio. Além disso, todos esses fatores, quando ocorridos na adolescência, predispõem a negligência com os futuros filhos desses adolescentes. Assim se estabelece um ciclo entre fatores de risco e comorbidades na adolescência.

Os picos de tentativas de suicídio ocorreram no inicio da adolescência (11 anos) e final dessa fase (25 anos). De acordo com a literatura, isso já era o esperado porque estas são as fases de maior transição e modificação na vida do adolescente (KRUG et al., 2002). Esse fator de risco tem implicações futuras na vida destes adolescentes, já que aumenta o risco de recidivas. Além disso, verifica-se que uma instabilidade familiar provocadas por problemas psiquiátricos e uma historia familiar positiva para suicídio nos pais pode ser um fator de risco para os filhos, perpetuando o ciclo (KRUG et al., 2002).

Existem fatores específicos para o suicídio que são: uma tentativa de suicídio prévio, distúrbios do 
humor (incluindo depressão), abuso de substâncias psicoativas, idade de 16 anos ou mais, homem e vivendo sozinho, história de abuso físico e sexual (ZAMETKIN; ALTER; YEMINI, 2001).

Um ambiente familiar em que há separação ou divórcio dos pais, mau relacionamento entre os pais e abuso sexual na infância são fatores ambientais que aumentam o risco dos adolescentes a tentar o suicídio (BEAUTRAIS, 2003).

Há que se considerar que o impacto de experiências adversas em vida precoce predispõe a aumentar a ansiedade e depressão na vida adulta e maior sensibilidade a resposta de estresse pela ativação do eixo hipotálamo-hipófise-adrenal e pelo aumento da secreção do hormônio corticotrofina (CRH) (HEIN; NEMEROFF, 1999).

O presente estudo verificou um aumento brusco na freqüência de DST dos 15 para os 16 anos e uma posterior estabilização dos 17 aos 25 anos. Esse aumento aos 16 anos era esperado, pois na faixa etária dos 15 aos 19 anos há maior negligência em relação ao uso dos preservativos(TAQUETTE; VILHENA; PAULA, 2004).

Os mesmos autores acima descrevem os resultados em seu estudo realizado com 356 adolescentes que tiveram DSTs. Mostraram que os rapazes têm maior número de parceiras e iniciam atividade sexual mais cedo. As moças, por sua vez, usam menos preservativos e são vítimas mais freqüentes de abuso sexual.

Taquette, Vilhena e Paula (2004) ressaltam ainda que nos casos de HIV houve um aumento significativo da faixa dos 10 aos 16 anos para a faixa dos 17 aos 25 anos, na qual a ocorrência desta última está em 90,32\%. O risco de HIV também está associado ao não uso dos preservativos que ocorre principalmente entre 15 e 19 anos.

Verificou-se um início mais precoce de depressão na adolescência, já com o registro de casos entre 10 e 16 anos. Além disso, adolescentes depressivos estão associados a danos psicossocias significativos e suicídio. Por outro lado, existe uma significativa morbidade e mortalidade em potencial para suicídio na fase adulta, cujo episódio depressivo ocorreu na infância (WEISSMAN et al., 1999). Entre os fatores de risco para depressão encontram-se também o abuso sexual e negligência na infância, perpetuando o ciclo depressão, maus tratos, depressão.

Autores como McCauley et al (1997) apontam que o abuso físico ou sexual na infância está associado com problemas de saúde na adolescência e na vida adulta, incluindo tentativas de suicídio, depressão, sintomas físicos, problemas psicológicos e abuso de substâncias psicoativas.

No presente estudo, foi verificado um aumento significativo de casos de gravidez dos 13 aos 16 anos. O aumento da gravidez nesta faixa etária reflete baixa auto-estima, cuja ocorrência é mais freqüente nas camadas sócio-econômicas mais baixas da população (SABROZA et al., 2004).

A gravidez no início da adolescência tem implicações futuras para a criança, pois a instabilidade da situação também acaba por contribuir para a ocorrência de prejuízos emocionais, e até mesmo de transtornos de ordem afetiva, muitas vezes agravados por um ambiente familiar pouco acolhedor e muito mobilizado pela notícia da gestação. O risco dos filhos serem vítimas de maus tratos é maior, principalmente nos casos em que a gravidez foi indesejada (SABROZA et al., 2004).

A violência nas relações afetivas dificulta a prevenção de DSTs /AIDS freqüentes na adolescência. A vivência de situações de opressão e constrangimento presentes nas idades de 10, 14, e 16 anos foram preditivas de violência em idades superiores aos 18 anos. Ser exposto à violência no contexto social, parece ter relação com vitimização e perpretação de atos violentos em outros contextos como o familiar e o interpessoal (RUZANY et al., 2003).

No futuro, outras pesquisas poderão avaliar fatores de risco como abuso sexual e físico e negligência na infância, contribuindo para a gravidez 
indesejada, DST/AIDS, depressão e tentativa de suicídio na adolescência e início da adultez.

\section{Conclusão}

O presente estudo demonstrou uma forte correlação entre gravidez, DST, HIV e depressão. Verificou-se que as condutas de risco iniciam-se cada vez mais precocemente, já por volta da idade de 10 anos, devido à imaturidade e instabilidade emocionais dos adolescentes. Tais condutas incluem o não uso de preservativos que, combinado com a difusão da sexualidade cada vez mais precoce, contribui para o aumento da gravidez, as DSTs, o HIV e a depressão. Infere-se, também, que a gravidez predispõe ao desencadeamento de repercussões emocionais negativas, principalmente quando não é planejada e amparada.

Ressalta-se o aumento significativo na freqüência de diagnósticos de depressão, gravidez, tentativas de suicido, DST e HIV nas idades de 17 a 25 anos (fase final da adolescência e adulto jovem).

Sugere-se uma intervenção que privilegie a autoestima e a prevenção através da educação e detecção precoce, que anteceda o ensino médio. A intervenção deverá ocorrer no ensino fundamental, para prevenir morbidade e mortalidade na adolescência e início da adultez. Para isso, é preciso identificar alternativas para promover a saúde e prevenir comportamentos de risco, motivando os adolescentes a desenvolver habilidades para a vida, tomada de decisões, habilidades interpessoais, aprendizado cooperativo e manejo de situações adversas.

Além disso, deve-se avaliar também uma forma de prevenção dos fatores ambientais relacionados ao abuso sexual e negligência na infância, que contribuíram para o surgimento de condutas de risco na adolescência.

Assim, a prevenção e intervenção precoce dos principais problemas da adolescência são fatos que interessam a toda sociedade, visto que esses indivíduos se constituirão em população adulta e produtiva.

\section{Colaboradores}

O Planejamento do estudo foi responsabilidade de Sandra Odebrecht Vargas Nunes e Rosimeire Abreu. A coleta, análise e discussão de dados foram conjuntos de S.O. V. Nunes; R. Abreu; A. L. Hirata; M. V. A. Nunes; L. R. Barbosa A. Terzis e R. M. Franco .

\section{Agradecimentos}

À Coordenadoria de Pós Graduação da Universidade Estadual de Londrina.

\section{Referências}

BAHLS, S. C. Epidemiologia de sintomas depressivos em adolescentes de uma escola pública em Curitiba, Brasil. Revista Brasileira de Psiquiatria, São Paulo, v.24, n.2, p.63-67, 2002.

BEAUTRAIS, A. Suicide and serious suicide attempts in youth: A multiple group comparison study. American Journal of Psychiatry, Washington, v. 160, n. 6, p. 1093 1099, 2003.

BLANKSON, M.; CLIVER, S. P.; GOLDENBERG, R. L.; HICKEY, C. A.; JIN, J.; DUBARD, M. B. Healthy behavior and outcomes in sequential pregnancies of black and white adolescents. Journal of the American Medical Association, Chicago, v.269, n.11, p.1401-1403, 1993.

CREATSAS, G.; GOUMALATSOS, N.; DELIGEOROGLOU, E.; KARAGITSOU, T.; CALPAKTSOGLOU, C.; AREFETZ, N. Teenage pregnancy: comparison with two groups of older pregnant women. Journal of Adolescent Health, New York, v.2, n.1,p. 77-81, 1991.

DUARTE, A. Gravidez na adolescência. Rio de Janeiro: Rosa dos Tempos, 1998.

FRIEDMAN, S. B.; PHILLIPS, S. Psychosocial risk to mothers and child as a consequence of adolescent pregnancy. Seminars in Perinatology, New York, v.5, n.1, p.33-37, 1981.

HEIN, C.; NEMEROFF, C. The impact of early adverse experiences on brain systems involved in pathophysiology of anxiety and affective disorders. Biological Psychiatry, New York, v.46, n.12, p.1509-1522, 1999. 
HIRSCHFELD, R. M. A.; RUSSELL, J. M. Assessment and treatment of suicidal patients. New England Journal of Medicine, Boston, v.337, n.13, p.910-915, 1997.

KRUG, E. G.; DAHLBERG, L. L.; MERCY, J. A.; ZWI, A. B.; LOZANO, R. Child abuse and neglect by parents and other caregivers. In:. WORLD HEALTHORGANIZATION. World report on violence and health. Geneva, 2002. p.59-81

LEWINSOHN, P. M.; ROHDE, P.; SEELEY, J. R; KLEIN, D. N; GOTLIB, L. H. Natural course of adolescent major depressive disorder in a community sample: predictors of recurrence in young adults. American Journal of Psychiatry, Washington, v.157, n. 10, p.1584-1591, 2000.

MARTIN, A.; DONALD, C. Adolescent depression: window of (missed?) opportunity. American Journal of Psychiatry, Washington, v.157, n.10, p.1549-1551, 2000.

MCCAULEY, J; KERN, D. E.; KOLODNER, K.; DILL, L.; SCHROEDER, A. F.; DECHANT, H. K; RYDEN, J.; DEROGATIS, L. R; BASS, E. B. Clinical characteristics of woman with a history of childhood abuse unhealed wounds. Journal of the American Medical Association, Washington, v.277, n.17, p.1362-1368, 1997.

MILLSTEIN, S.; NIGHTINGALE, E. O; PETERSEN, A.C.; MORTIMER, A. M.; HAMBURG, D.A. Promoting the healthy development of adolescents. Journal of the American Medical Association, Chicago, v.269, n.11, p.1413-1415, 1993.

OMS. Necesidades de salud de los adolescentes. Genebra, 1977.

PIYASIL, V. Anxiety and depression in teenage mothers: a comparative study. Journal of the Medical Association of Thailand, Bangkok, v.81, n.2, p.125-129, 1998.
RUZANY, M. H.; TAQUETTE, S. R.; OLIVEIRA, R. G.; MEIRELLES, Z. V.; RICARDO, I. B. A violência nas relações afetivas dificulta a prevenção de DST/AIDS? Jornal de Pediatria, Rio de Janeiro, v.79, n.4, p.349-354, 2003.

SABROZA, A. R.; LEAL, M. C.; SOUZA JÚNIOR, P. R.; GAMA, S. G. N. Algumas repercussões emocionais negativas da gravidez precoce em adolescentes do município do Rio de Janeiro (1999 -2001). Cadernos de Saúde Pública, Rio de Janeiro, v. 20, Supl.1, p.S130-S137, 2004.

TAQUETTE, S.; VILHENA, M.; PAULA, M. Doenças sexualmente transmissíveis e gênero: um estudo transversal com adolescentes no Rio de Janeiro. Cadernos de Saúde Pública, Rio de Janeiro, v.20, n.1, p.282-290, 2004.

WAGNER, D. K.; BERENSON, A.; HARDING, O.; JOINER, T. Attributional style and depression in pregnant teenagers. American Journal of Psychiatry, Washington, v.155, n. 9, p.1227-1233, 1998.

WEISSMAN, M. M. Wolk S, Goldstein RB, Moreau D, Adams P, Greenwald S. Adolescentes deprimidos como ficam quando adultos. Journal of the American Medical Association, Rio de Janeiro, v.3, n.9, p.2429-2435, 1999. (Ed. port.).

WILCOX, H.; FIELD, T. Correlations between the BDI and CES-D in a sample of adolescent mothers. Adolescence, Roslym Heights, v.33, n.131, p.565-574, 1998.

WITTCHEN, H.U.; NELSONC, B.; LACHNER, G. Prevalence of mental disorders and psychosocial impairments in adolescents and young adults. Psychological Medicine, London, v.28, n.1, p.109-126, 1998.

ZAMETKIN, A.; ALTER, M.; YEMINI, T. Suicide in teenagers assessment, management and prevention. Journal of the American Medical Association, Washington, v.286, n.24, p.3120-3125, 2001. 